\title{
Evaluation of Educational Advancement following a Sports Medicine Rotation
}

\author{
Edward Rozek, PhD', Allison Cracchiolo, BS ${ }^{1}$, Patrick Keating, $B S^{1}$, Kate Hefferan, $B S^{1}$, \\ Chaoyang Chen, $M D^{2}$ and Stephen Lemos, MD, PhD $^{1^{*}}$
}

${ }^{1}$ Department of Orthopaedic Surgery and Sport Medicine, Detroit Medical Center, USA

${ }^{2}$ Department of Biomedical Engineering, Wayne State University, USA

*Corresponding author: Stephen Lemos, MD, PhD, Sports Medicine Fellowship Program Director, Department of Orthopaedic Surgery and Sport Medicine, Detroit Medical Center, 3990 John R Street, Box 137, Detroit, MI 48201, USA, Tel: 313-745-0539, Fax: 313-745-1545

\section{Abstract}

Background: The competency in orthopaedic surgery and sports medicine has been reported as a deficiency. Sports medicine clinical rotations may improve both medical students' and residents' musculoskeletal knowledge, but none have evaluated the knowledge and skills a resident may gain during the rotation in sports medicine.

Objective: The purpose of this study is to determine (1) The knowledge gained by residents at varying levels after rotating within our sports medicine program and (2) The performance of residents in a standardized sports medicine test at the start and end of each resident's rotation in sports medicine.

Methods: Total of 136 residents and fellows participated in this study following the Model Curriculum and Guidelines for Orthopaedic Surgery Sports Medicine Fellowship Training created by the American Orthopaedic Society for Sports Medicine Accreditation Council of Graduate Medical Education. Multiple modalities training program were administered, including clinical rotation, laboratory research project, covering professional sport team training, and conference presentation. Their performances were assessed and compared by pre- and post-rotation tests with statistical analysis to determine the improvement of their orthopaedic knowledge.

Results: The residents at the fifth of Post-Graduate-Year (PGY 5) scored the highest followed by fellows. PGY5 had the lowest percent change between pre- and post-test. PGY 2 and PGY3 residents scored the lowest overall while having the largest percent change before and after the rotation thereby showing the most improvement.

Conclusions: Multiple modalities training program improved residents and fellows' performance and knowledge in sports medicine.
\end{abstract}

\section{Introduction}

The musculoskeletal complaint is one of the most common reasons for visiting a physician and accounts for 92.1 million cases annually [1] with approximately $30 \%$ of visits to primary care physicians and $20 \%$ of visits to the Emergency Department (ED) [2,3]. Despite the prevalence of musculoskeletal disorders, competency in musculoskeletal medicine including sports medicine has still been reported as a deficiency in medical education in the USA [4-9]. This deficiency is well documented through studies at both the undergraduate and graduate medical education levels $[7,8,10-12]$. This deficiency is noted even among orthopedic surgery residents [12]. Residents have failed to demonstrate basic competency in musculoskeletal medicine, revealing medical school preparation regarding this topic is inadequate. Because of this deficiency of knowledge and skills at all levels of medical education, more than half of residents did not feel they had adequate training in musculoskeletal medicine [13].

It has been reported that sports medicine clinical rotations improve both medical students' and residents' musculoskeletal knowledge [7,14-17] but none have tried to evaluate the knowledge and skills a resident may gain during his/her rotation in sports medicine. We hypothesized that in addition to look at the predictors of residency, or success on orthopaedic surgery board examinations as showed in the previous studies [7,1417 ] it is necessary to quantify the knowledge and skills

Citation: Rozek E, Cracchiolo A, Keating P, Hefferan K, Chen C, et al. (2021) Evaluation of Educational Advancement following a Sports Medicine Rotation. Int J Sports Exerc Med 7:196. doi.org/10.23937/24695718/1510196

Accepted: July 17, 2021; Published: July 19, 2021

Copyright: (C) 2021 Rozek E, et al. This is an open-access article distributed under the terms of the Creative Commons Attribution License, which permits unrestricted use, distribution, and reproduction in any medium, provided the original author and source are credited. 
that a resident may gain during his/her various sports medicine rotations. It is important to identify a body of core orthopaedic knowledge, specific goals and expectations, and common benchmarks to measure program effectiveness. The purpose of this study is to assess the knowledge gained by residents' rotating within our sports medicine program at varying levels. A standardized sports medicine test was administered at the start and end of each resident's rotation. The pre- and post-rotation scores were juxtaposed for each resident as well as each level of training.

\section{Methods}

\section{Educational methods}

The Sports Medicine (SM) rotation programs at DMC were developed based upon the Model Curriculum and Guidelines for Orthopaedic Surgery Sports Medicine Fellowship Training created by the American Orthopaedic Society for Sports Medicine Accreditation Council of Graduate Medical Education [18]. This model curriculum was designed to be used as a resource and guide in developing curriculum for sport medicine residency rotations and fellowship programs. These documents include a comprehensive list of specific goals and objectives for core content material, including orthopedics and prioritizing content items, to indicate the depth and breadth of knowledge required of a specialist in SM. The knowledge and performance at appropriate PGY skill level in both cognitive and technical skills were evaluated after rotations, The assessments included: 1) The ability to manage patients with common musculoskeletal problems in an outpatient clinic, 2) The ability to organize patients' history and physical examinations, 3) A differential diagnosis for common problems including pain in musculoskeletal system, 4) The special skill necessary for a sport team coverage, 5) The ability to be part of the health care team with coaches, players and trainers, 6) Performance of arthroscopic knee and shoulder exams accurately and in a timely manner, 7) Reading and understanding the imaging used in patient care sports medicine.

\section{Participants and clinical rotations}

Total 136 residents and fellows participated in this study, including 4 of PGY2s, 71 of PGY3s, 32 of PGY5s, and 29 of fellows. Resident and fellows rotated among different hospitals at Detroit metropolitan area. Hospitals included Detroit Medical Center, Henry Ford Wyandotte, Oakwood Hospital \& Chelsea Community Hospital, Unasource Hospital, Premier and Waterford Surgery Centers, Henry Ford Macomb, McLaren Macomb, William Beaumont Hospital at Grosse Pointe, McLaren Oakland Hospitals. Residents rotated with sports medicine for three months, a group of external residents rotated for two months, and fellows rotated for one year.

\section{Professional and amateur team coverage}

Residents and fellows worked with physicians covering games for professional teams including the Detroit Tigers (MLB) and the Detroit Red Wings (NHL). In addition to game coverage, SM fellows also travelled to and participated in spring training, training camps, and pre-season physicals. They worked with physicians to cover sport events and outreach including the Detroit Free Press Marathon, Detroit Grand Prix and other events sponsored by DMC Sports Medicine. They also worked with physician to cover amateur high school teams throughout the year.

\section{Academic conference presentations}

Residents and fellows presented academic lectures at weekly fellow conferences, as well as case presentations at the monthly DMC Sports Medicine Visiting Lecture Series and monthly Journal Clubs. They attended orthopaedic surgery resident conferences and other local sports medicine orthopaedic surgery meetings. All Sports Medicine Fellows attended the annual American Orthopaedic Society for Sports Medicine (AOSSM) Conference as well as the Michigan Orthopaedic Society Fall and Spring conferences. Sports Medicine Fellows also attended various arthroscopy courses locally, regionally, and nationally. All Sports Medicine Fellows presented their research findings and attended a two-day event in July hosting speakers from the Metro Detroit area and national Keynote Speakers. All DMC Sports Medicine Orthopaedic Fellowship alumni, present fellows, and incoming fellows were invited as a way for fellows to stay connected with each other and the Program.

\section{Training in laboratory settings}

Residents and fellows attended weekly Bioskills Labs focusing on improving arthroscopic skills of the shoulder, elbow, wrist, hip, knee, and ankle. There was also a musculoskeletal ultrasound component to the Bioskills Lab for training. A 12-week rotating schedule in the Bioskills Lab ensured that fellows participated and gained mastery of skills which enables the fellows to teach the lab for residents and students during the last quarter of the Fellowship year.

\section{Research projects}

Each Sports Medicine Fellow is asked to complete one research project during their tenure. Fellows are also requested to initiate a project for the incoming fellow for the following year to ensured that all fellows participated in 2 research projects during their fellowship. Research curriculum included clinical studies [19-23] traditional biomechanical studies [24-26] and emerging machine learning Artificial Intelligence (AI) techniques in clinical translational research [27-29].

\section{Assessments of residents and fellows' performance}

To assess knowledge gained during a sports medicine rotation, standardized sports medicine test was used. 


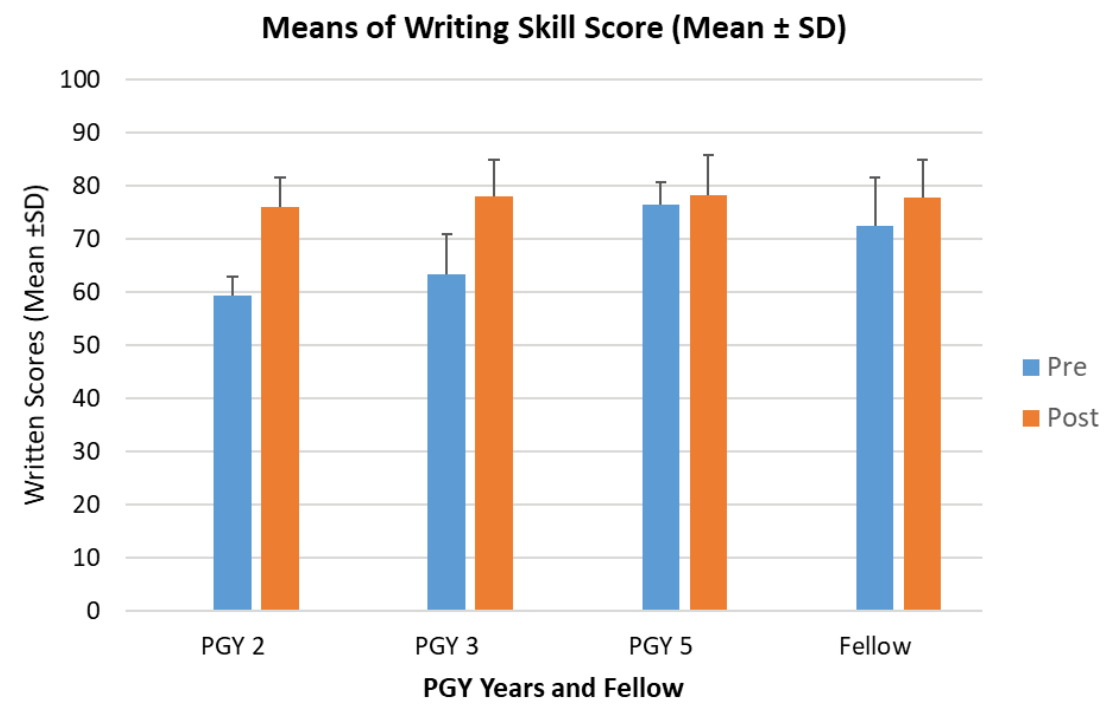

Figure 1: Mean writing scores of residents and fellows.

This examination was designed to identify strengths and weaknesses in the clinical and practice management areas of orthopaedic sports medicine. The pre-test was performed within the first week of the rotation for residents entering their third and fifth year; as well as fellows. The post-test was performed the final week of the residency rotation and fellowship year. Incorrect answers were revisited with follow-up short answer questions written by board-certified physician assistants. Examination scores were recorded for both pre- and post-tests. The average, standard deviation, and percent change were calculated for each group and compared.

\section{Statistical analysis}

One-Way ANOVA and GLM Univariate with PostHoc LSD tests were performed to determine the significance of difference between different years of training for residents and fellow regarding to their testing scores. SPSS software (IBM, Version 25, Armonk, NY) was used for statistical analysis.

\section{Results}

For writing skill in all four groups (PGY-2, PGY-3, PGY-5, and fellow) tested, there was significant overall improvement between pre- and post-test scores (GLM, Univariate PostHoc LSD, $p=0.000$ ). The pre-test writing skill scores of PGY 5 and follows were higher than the scores of PGY 2 and PGY 3 (ANOVA, PostHoc LSD, $p=$ $0.006)$. There was not statistical difference of posttest writing skill between PGY 2 and PGY 3, as well as between PGY 5 and fellow (ANOVA, PostHoc LSD, $p=$ 0.307) (Figure 1).

Pre-test scores ranged from 44.00-76.00 for PGY3, 68.00-83.20 for PGY5, and 59.20-83.20 for fellows. Posttest scores ranged from $60.80-83.20,72.80-87.20$, and 70.40-92.00. The percent changes were $28.4 \%, 23.3 \%$, $2.3 \%$, and $7.3 \%$. The fellow group achieved the highest pre- and post-test scores on this standardized test model, while the PGY2 year achieved the largest percent change $(28.4 \%)$ followed by PGY3 $(23.3 \%)$ between the pre- and post-tests.

For knee knowledge in all four groups (PGY-2, PGY-3, PGY-5, and fellow) tested, there was significant overall improvement between pre- and post-test scores (GLM, Univariate PostHoc LSD, $p=0.003$ ). The knee knowledge pre-test score of PGY 5 was higher than the scores of PGY 2 and PGY 3 (ANOVA, PostHoc LSD, $p=0.03$ and $p=$ 0.002 respectively). There was not statistical difference of knee knowledge post-test score between 4 groups (ANOVA, PostHoc LSD, $p>0.117$ ) (Figure 2).

For shoulder knowledge in all four groups (PGY-2, PGY-3, PGY-5, and fellow) tested, there was significant overall improvement between pre- and post-test scores (GLM, Univariate PostHoc LSD, $p=0.006$ ). The shoulder knowledge pre-test score of PGY 5 was higher than the scores of PGY 2 and PGY 3 (ANOVA, PostHoc LSD, $p=0.018$ and $p=0.000$ respectively). The shoulder knowledge pre-test score of fellows was not significantly higher than the scores of PGY 2 and PGY 3 (ANOVA, PostHoc LSD, $p=0.124$ and $p=0.071$ respectively). There was not statistical difference of knee knowledge post-test score between 3 groups including (PGY 3, PGY 5, and fellow) (ANOVA, PostHoc LSD, $p>0.885$ ). The shoulder knowledge post-test score of PGY 2 was not significantly lower than other 3 groups (ANOVA, PostHoc LSD, $p=$ $0.028, p=0.038, p=0.032$ respectively) (Figure 3 ).

\section{Discussion}

This study demonstrated that residents and fellows gained knowledge within the sports medicine field train after rotation. The groups with the most experience and post-graduate training (PGY 5 and fellow) scored the highest overall, with the lowest percent change between pre- and post-test. Conversely, the group with the least experience and post-graduate training (PGY 2 and 3) scored the lowest overall while having 


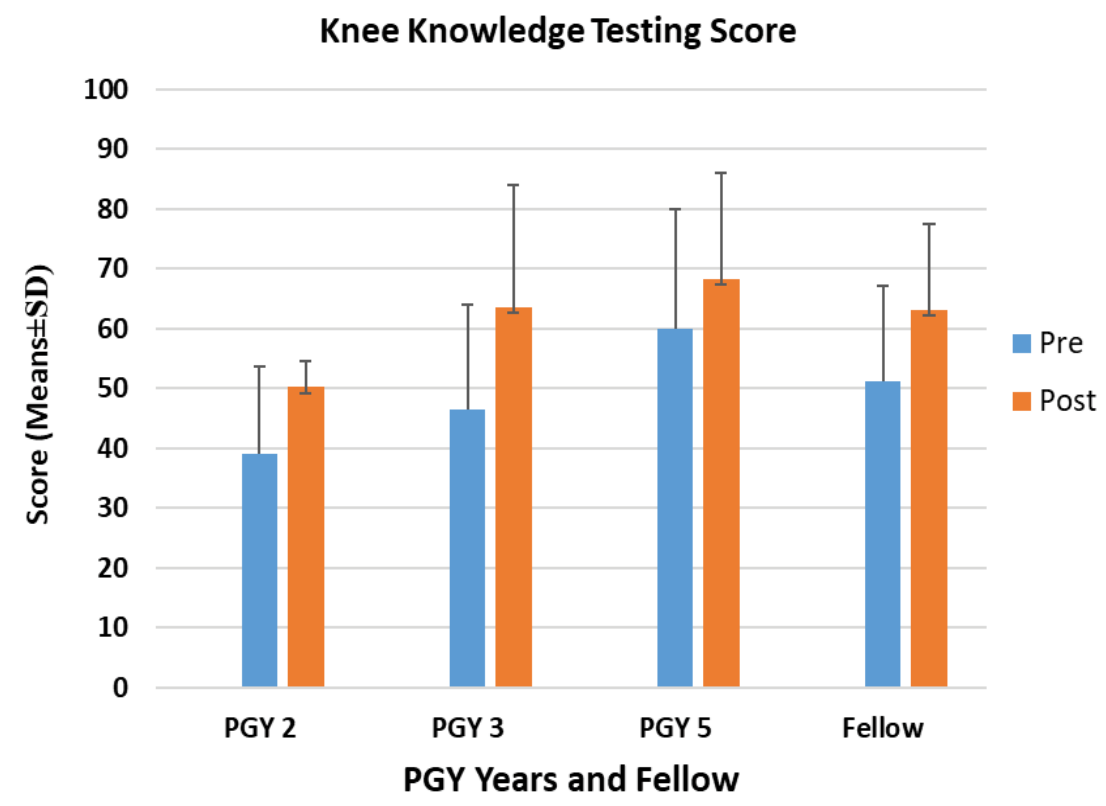

Figure 2: Knee knowledge scores of residents and fellows.

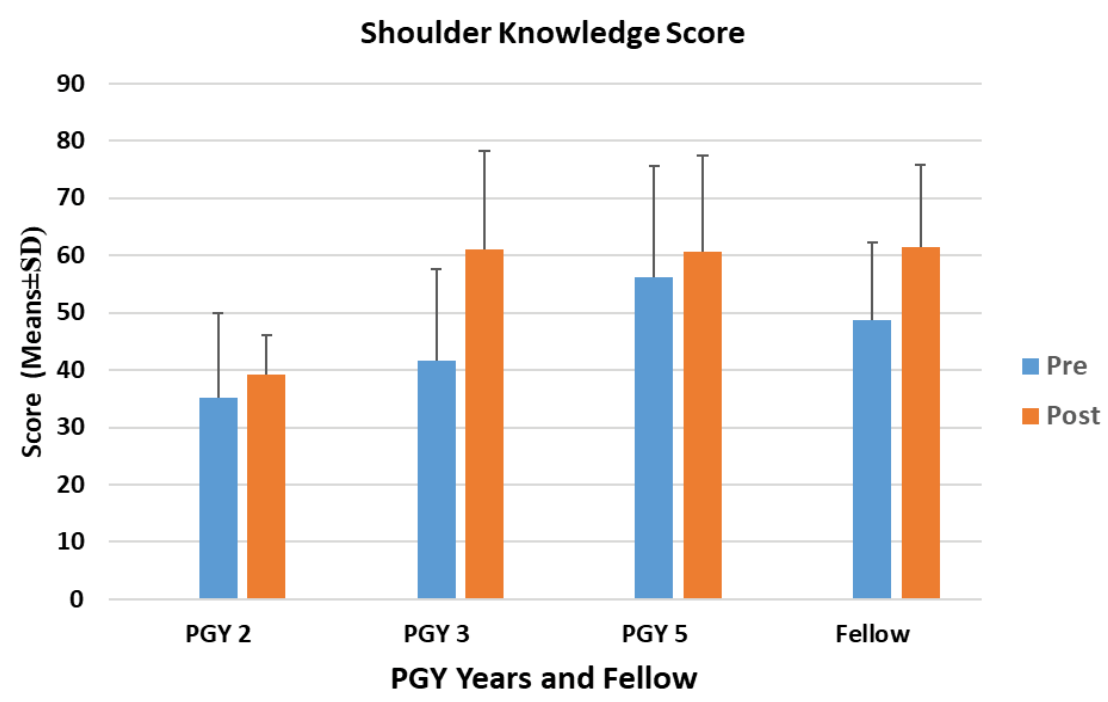

Figure 3: Shoulder knowledge testing scores of residents and fellows.

the largest percent change thereby showing the most improvement. The groups followed a trend of showing stepwise improvement with additional years of postgraduate education and training. This information from this study has now been utilized to establish other avenues to quantify skills and knowledge attained during our sports medicine rotation, including knowledge on the elbow, hip, foot, and ankle.

The introduction of an objective sports medicine rotation test has been very well received by our DMC orthopaedic surgery residents and fellows. DMC residents on the sports medicine rotation had a statistically significant higher rate of satisfaction of their educational experience when compared to other traditional orthopedic rotations. Residents' perception of the quality of their education while on sports medicine was consistently high on the sports medicine rotation, evidenced by improvement of their knowledge testing score after rotation. We believe instituting pre- and post-testing for the sport medicine rotation has provided the residents and fellows an objective way to measure their knowledge, improvement, and satisfaction during the rotation. Post-rotation evaluations also showed the residents and fellows are happy with the overall organization and commitment of the faculty and staff to help them improve skills.

In DMC Sports Medicine training, residents took an in-service training examination yearly and both residents and fellows were ultimately required to pass their orthopaedic board-certification, we believe the sports medicine rotation exams help the residents and fellows prepare for this examination. We are also currently working on a project to formally evaluate the core content musculoskeletal knowledge and physical examination skills of our current residents and fellows to further enhance the rotation. 
Our experience suggests that the implementation of some type of pre- and post-testing assessment is a practical and useful way for residents and fellows to assess objective knowledge and skills developed during a rotation cycle that also improves overall satisfaction of the rotation experience.

We will continue to evaluate and improve our testing and assessment methods focusing on how to best achieve and evaluate clinical competency in sports medicine and musculoskeletal medicine.

\section{Limitations}

This study was performed at a single institution and, therefore, may not be generalizable to other programs. Future studies include multiple-center collaborative investigation to optimize the resident's training curriculum.

\section{Conclusion}

Multiple modalities training program, inducing clinical rotation, laboratory research project, participating in covering professional sport team training, and conference presentation, improved residents and fellows' performance in sports medicine. Our experience suggests that the implementation of this modalities training program is an effective approach orthopedic and sport medicine resident and fellow training. DMC orthopaedic residents' evaluation of the sports medicine experience has remained positive and has a statistically significant higher rate of performance improvement.

\section{Acknowledgments}

Authors would like to thank Rachel Toccalino, Linda Gross assistance in this research.

\section{Disclosure}

The authors report no conflicts of interest in this work.

\section{References}

1. Sweeney CL, Davidson M, Melgar T, Patel D, Cucos D (2003) The current status of sports medicine training in United States internal medicine residency programmes. $\mathrm{Br}$ J Sports Med 37: 219-225.

2. Washington DL, Shekelle PG, Stevens CD (2001) Deferred care for adults with musculoskeletal complaints. Eff Clin Pract 4: 65-72.

3. De Lorenzo RA, Mayer D, Geehr EC (1990) Analyzing clinical case distributions to improve an emergency medicine clerkship. Ann Emerg Med 19: 746-751.

4. Booth A, Wise DI (1990) General practice training in musculoskeletal disorders. Br J Gen Pract 40: 390.

5. Freedman KB, Bernstein J (1998) The adequacy of medical school education in musculoskeletal medicine. J Bone Joint Surg Am 80: 1421-1427.

6. Freedman KB, Bernstein J (2002) Educational deficiencies in musculoskeletal medicine. J Bone Joint Surg Am 84:
604-608.

7. Schmale GA (2005) More evidence of educational inadequacies in musculoskeletal medicine. Clin Orthop Relat Res 437: 251-259.

8. Skelley NW, Tanaka MJ, Skelley LM, LaPorte DM (2012) Medical student musculoskeletal education: an institutional survey. J Bone Joint Surg Am 94: 141-147.

9. Sabesan VJ, Schrotenboer A, Habeck J, Lombardo D, Stine S, et al. (2018) Musculoskeletal Education in Medical Schools: A Survey of Allopathic and Osteopathic Medical Students. J Am Acad Orthop Surg Glob Res Rev 2: 019.

10. Matzkin E, Smith EL, Freccero D, Richardson AB (2005) Adequacy of education in musculoskeletal medicine. $J$ Bone Joint Surg Am 87: 310-314.

11. DiCaprio MR, Covey A, Bernstein J (2003) Curricular requirements for musculoskeletal medicine in American medical schools. J Bone Joint Surg Am 85: 565-567.

12. Beran MC, Awan H, Rowley D, Samora JB, Griesser MJ, et al. (2012) Assessment of musculoskeletal physical examination skills and attitudes of orthopaedic residents. $J$ Bone Joint Surg Am 94: e36.

13. Hing E, Cherry DK, Woodwell DA (2006) National Ambulatory Medical Care Survey: 2004 summary. Adv Data 1-33.

14. Day CS, Yeh AC, Franko O, Ramirez M, Krupat E (2007) Musculoskeletal medicine: an assessment of the attitudes and knowledge of medical students at Harvard Medical School. Acad Med 82: 452-457.

15. DiGiovanni BF, Southgate RD, Mooney CJ, Chu JY, Lambert DR, et al. (2014) Factors impacting musculoskeletal knowledge and clinical confidence in graduating medical students. J Bone Joint Surg Am 96: 185.

16. Dwyer T, Theodoropoulos JS, Herold J, Henry P, Wasserstein D, et al. (2013) Assessing competence of orthopaedic residents: the reliability and validity of an objective structured clinical examination after a sports medicine rotation. J Bone Joint Surg Am 95: e177.

17. Watts SA, Zhang Z (2011) Competency in musculoskeletal and sports medicine: Evaluating a PGY-1 curriculum. Fam Med 43: 659-663.

18. AAOS. American Orthopaedic Society for Sports Medicine AOSSM Educational Curriculum.

19. Lane MK, Mutch J, Ratkowiak K, Lemos SE, Kalra K, et al. (2017) Proximity of Lateral Critical Structures to the All-Epiphyseal Outside-In Femoral Tunnels in Pediatric Anterior Cruciate Ligament Reconstruction. Arthroscopy 33: $1234-1240$.

20. Schafer P, Mehaidli A, Zekaj M, et al. (2020) Assessing knee anatomy using Makoplasty software a case series of 99 knees. J Orthop 20: 347-351.

21. Hamilton DA, Ononuju U, Nowak C, Chen C, Darwiche H (2021) Differences in Immediate Postoperative Outcomes Between Robotic-Assisted TKA and Conventional TKA. Arthroplast Today 8: 57-62.

22. Yokhana SS, Hamilton DA, Stine SA, Stimson LN, Zalikha AK, et al. (2021) The prevalence of posterolateral tibial baseplate overhang: An anatomical CT study. J Orthop 23: 259-263.

23. Schultz K, Osborne J, Nelson K, Potini V, Chen C, et al. (2020) Intra- and interobserver reliability for predicting hip preservation versus hip arthroplasty utilizing plain 
radiographs with comparison of surgeon specialization. J Hip Preserv Surg 7: 70-76.

24. Mancini EJ, Kohen R, Esquivel AO, Cracchiolo AM (2017) Comparison of ACL Strain in the MCL-Deficient and MCL-Reconstructed Knee During Simulated Landing in a Cadaveric Model. Am J Sports Med 45: 1090-1094.

25. Lissy M, Osborne J, Keating P, Richards N, Chen C, et al. (2021) Physeal-sparing posteromedial portal approach reduced distance between guide pin and neurovascular structures. Knee Surg Sports Traumatol Arthrosc 29: 881888.

26. Doig T, Fagan P, Frush T, Lovse L, Chen C, et al. (2020) The all-inside all-suture technique demonstrated better biomechanical behaviors in meniscus radial tear repair. Knee Surg Sports Traumatol Arthrosc 28: 3606-3612.
27. Chen C, Chen C, Mei X, Chen C, Ni G, et al. (2019) Effects of Image Augmentation and Dual-layer Transfer Machine Learning Architecture on Tumor Classification. 2019: 282287.

28. Cooke C, Osborne J, Jackson N, Keating P, Flynn J, et al. (2020) Acetaminophen, bupivacaine, Duramorph, and Toradol: A comparison of chondrocyte viability and gene expression changes in osteoarthritic human chondrocytes. Knee 27: 1746-1752.

29. Jiang Y, Chen C, Zhang X, et al. (2020) Shoulder muscle activation pattern recognition based on SEMG and machine learning algorithms. Comput Methods Programs Biomed 197: 105721 